\title{
Mir-26b inhibits growth and resistance to paclitaxel chemotherapy by silencing the CDC6 gene in gastric cancer
}

\author{
Bochao Zhao, Jiale Zhang, Xiuxiu Chen, Huimian Xu, Baojun Huang
}

Department of Surgical Oncology, First Affiliated Hospital, China Medical University, Shenyang, China

Submitted: 1 December 2016

Accepted: 23 February 2017

Arch Med Sci 2019; 15, 2: 498-503

DOI: https://doi.org/10.5114/aoms.2018.73315

Copyright $@ 2018$ Termedia \& Banach

\section{Abstract}

Introduction: Gastric cancer is one of the most common cancers of the digestive system and is associated with high morbidity and mortality. The aim of this study was to investigate whether miR-26b is involved in the proliferation and resistance to paclitaxel chemotherapy in gastric cancer cells.

Material and methods: The expression of miR-26b in gastric cancer cell lines was determined by quantitative real-time PCR. Bioinformatics software was used to predict potential target genes of miR-26b. Luciferase assay was used to verify the interactions between target genes and miR-26b. CDC6 protein expression was measured by Western blot. The proliferation and chemotherapy resistance were analyzed by MTT assay. Cell invasion was evaluated by Transwell assay.

Results: MiR-26b was down-regulated in gastric cancer cell lines compared to normal control cells, and its expression in drug resistance cells was even lower $(p<0.05)$. CDC6 was identified as a potential target gene of miR-26b by using bioinformatics analysis software. The expression of CDC6 was inhibited by miR-26b both at RNA level, which was determined by luciferase assay, and at protein level, which was determined by Western blot $(p<0.05)$. Silencing CDC6 inhibited cell proliferation, invasion, and promoted apoptosis of gastric cancer cell lines, BGC823 and SGC7901 ( $p<0.05)$. Moreover, CDC6 knockdown inhibited chemotherapy resistance to paclitaxel, IC50 to paclitaxel decreased from $153.17 \pm 0.49 \mu \mathrm{g} / \mathrm{l}$ to $39.81 \pm 0.28 \mu \mathrm{g} / \mathrm{l}(p<0.05)$. Conclusions: miR-26b inhibits growth and resistance to paclitaxel chemotherapy by silencing the CDC6 gene in the gastric cancer cell line SGC7901.

Key words: gastric cancer, miR-26b, CDC6, chemotherapy, paclitaxel.

\section{Introduction}

Gastric cancer (GC) is one of the most common malignant tumors in the digestive system, and is associated with high morbidity and mortality [1]. The prognosis of locally advanced GC is poor: 5-year survival remains in the $30-50 \%$ range despite the combined efforts of surgical resection, adjuvant chemotherapy and/or radiotherapy [2]. Therefore, there is an unmet need to develop novel therapeutic strategies to improve the outcome of GC.

MicroRNAs are a class of small non-coding RNAs, which possess various functions involving the regulation of apoptosis, proliferation, and autophagy [3-6]. MiR-26b (gene ID: 31612) belongs to the miR-26 fam-

\author{
Corresponding author: \\ Prof. Baojun Huang \\ Department of \\ Surgical Oncology \\ Affiliated First Hospital \\ China Medical University \\ 155 Nanjing North St \\ Heping District \\ 110001 Shenyang, China \\ Phone: +86 2422703576 \\ E-mail: \\ huangbaojun2014@163.com
}


ily. It has been found to be down-regulated in a variety of malignant tumors, including hepatocellular carcinoma, ovarian carcinoma and prostate cancer [7-9]. It has been hypothesized that miR-26b functions as a tumor suppressor gene in regulating apoptosis, cell differentiation, invasion, and radiosensitivity, likely by silencing its target genes [7-10]. However, little is known about the expression and functions of miR-26b in GC. In this study, we found that expression of miR-26b was downregulated in GC, and overexpression of miR$26 \mathrm{~b}$ inhibited growth and resistance to paclitaxel chemotherapy by silencing the CDC 6 gene in the gastric cancer cell line SGC7901. The findings may shed light on developing a potential novel therapeutic target of GC.

\section{Material and methods}

\section{Cell culture}

The human normal gastric epithelial cell line GES-1, GC cell lines BGC823 and SGC7901 and HEK293T cells were obtained from China Academy of Chinese Medical Sciences. The human GC SGC7901/PA cell line (resistance to paclitaxel) was obtained from the Fourth Military Medical University (Xi'an, Shanxi, China). These cells were cultured in RPMI-1640 medium containing 10\% fetal bovine serum (Gibco, Carlsbad, CA, USA), and cultured with $5 \% \mathrm{CO}_{2}$ at $37^{\circ} \mathrm{C}$. In order to maintain the sensitivity phenotype, SGC7901/PA cells was cultured with $20 \mu \mathrm{g} / \mathrm{l}$ paclitaxel (Gibco, Carlsbad, (A, USA), and cultured in paclitaxel-free medium for 14 days prior to the experiment.

\section{Quantitative real-time PCR (qRT-PCR)}

Total RNA was extracted with Trizol reagent and cDNA was synthesized with the High Capacity cDNA Reverse Transcription Kit (Applied Biosystems, Foster City, CA, USA). The RNA expression levels of miR-26b and cell division cycle 6 (CDC6) were quantified by SYBR (Applied Biosystems, Foster City, CA, USA). The primers for CDC6 were 5'-AACCACTGTCTGAATGTAAATC-3' (forward) and 5'-CTGTTACCATCAACTTCTGAG-3' (reverse). GAPDH and $U 6$ were used as endogenous reference genes [11].

\section{MicroRNA targets prediction and dual-luciferase reporter assay}

Target genes of miR-26b were predicted by miRanda (http://www.microrna.org) and TargetScan (http://www.targetscan.org). The wild-type and mutant-type luciferase reporter vectors (pmiRCDC6-wt and pmiR-CDC6-mut), constructed by the Genescript company (Nanjing, China), included the wild-type binding site and mutant binding site of CDC6 3'UTR. MiR-26b agonist (agomiR-26b) or negative control agonist (agomiR-NC) were synthesized by the Ribobio company (Guangzhou, China). AgomiR-26b is a chemically synthesized miRNA mimic, which was used to mimic the overexpression of miR-26b [12]. MicroRNA and luciferase reporter vector were cotransfected into HEK 293T cells. Luciferase reporter assay was performed as previously reported [13].

\section{Western blot}

Protein samples were extracted from cells and separated by polyacrylamide gel electrophoresis, transferred to PVDF membrane. The membrane was hybridized with CDC6 antibody (ab155759, Abcam, Cambridge, MA, USA) followed by secondary antibody. BeyoECL Plus reagent (Beyotime, Nanjing, China) was used to visualize the immunoblots, and the images were analyzed by ImageJ software (BD, Franklin Lakes, NJ, USA) to quantify the expression of $\mathrm{CDC} 6$ protein.

\section{RNA interference}

We designed RNAi vectors with three different targeting sites (pSilencer, ThermoFisher, Foster City, CA, USA). The vectors were constructed by Sangon Company (Shanghai, China). All three RNAi vectors were tested for the inhibition of the expression of CDC6. The vector that showed the best inhibitive effect was chosen for our experiment. The sequences of the nucleotides used for this vector were: 5'-GATCCCCCACTCTCCGAATGTAAATCTTCAAGAGAGATTTACATTCGGAGAGTGTTTTTTT-3' (sense) and 5'-AGCTAAAAAACACTCTCCGAATGTAAATCTCTCTTGAAGATTTACATTCGGAGAGTGGGG-3' (antisense). An empty pSilencer vector was used as a control (pS-NC).

\section{Cell proliferation and chemotherapy resistance assay}

Cellular proliferation was evaluated by MTT assay according to our previous study [14]. Cells were seeded into 96-well plates with 3000 cells per well and treated with paclitaxel $(10 \mu \mathrm{g} / \mathrm{l}$, $25 \mu \mathrm{g} / \mathrm{l}, 50 \mu \mathrm{g} / \mathrm{l}, 100 \mu \mathrm{g} / \mathrm{l}$ and $200 \mu \mathrm{g} / \mathrm{l})$ [15]. After $48 \mathrm{~h}$, the cellular viability was measured, and a dose-response curve was drawn to calculate the half maximal inhibitory concentration (IC50) using a Probit regression model.

\section{Cell invasion assay}

Cell invasion was measured using a Transwell chamber (Costar, Corning, NY, USA) with polycarbonic membrane $(6.5 \mathrm{~mm}$ in diameter, $8 \mu \mathrm{m}$ pore size) and Matrigel (BD, NJ, USA) as previously reported [16]. 


\section{Statistical analysis}

Statistical analysis was performed with the software SPSS 21.0 (IBM, Somers, NY, USA). The data were presented as mean \pm SD of five independent experiments. Student's $t$-test was used to analyze the difference between experiments. $P<0.05$ means a significant difference.

\section{Results}

The expression of miR-26b was down-regulated in GC cells

By using qRT-PCR, the miR-26b expression levels were found to be significantly lower in two independent GC cancer cell lines, BGC823 and SGC7901, compared to that in GES1 cells. In addition, the expression level of miR-26b was lower in SGC7901/PA cells than that in SGC7901 cells ( $p<$ 0.05 , Figure $1 \mathrm{~A})$. These findings suggest that miR$26 \mathrm{~b}$ may play a role in tumorigenesis and chemotherapy resistance of GC.

\section{MiR-26b functionally targets CDC6 in GC cell lines}

By using bioinformatics software, both TargetScan and miRanda identified a conserved miR-26b binding sites in the $3^{\prime}$-UTRs of CDC6 at the position of 109-115 bp (Figure $2 \mathrm{~A}$ ).
A

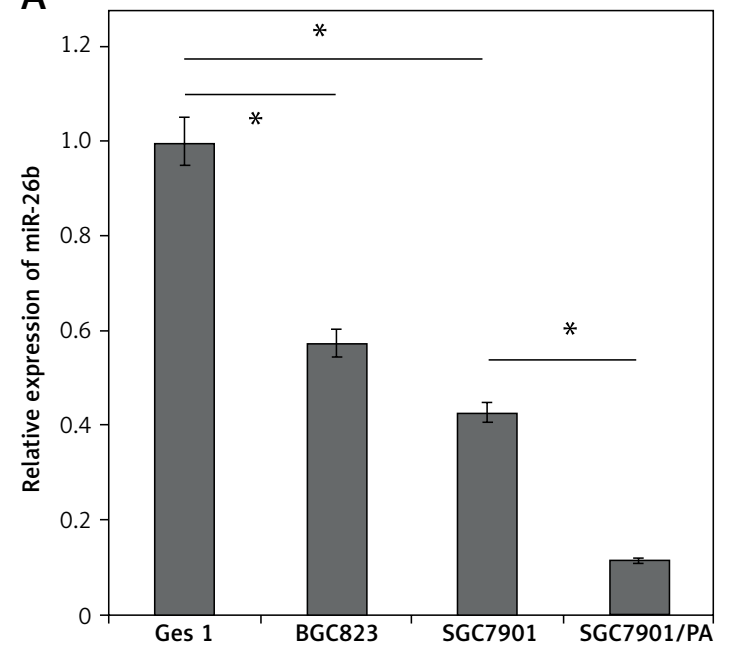

B

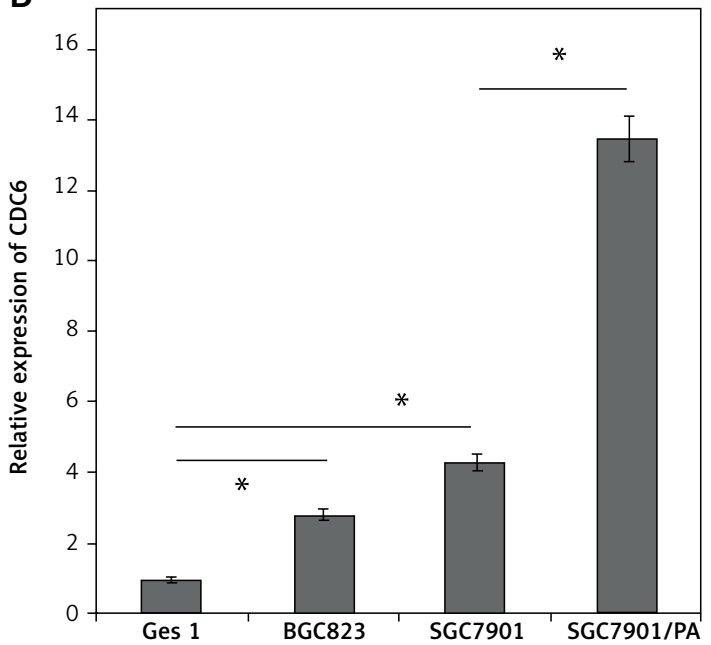

Figure 1. Expression of miR-26b and CDC6 genes in gastric cancer cell lines. A - Expression of miR-26b in BGC823 and SGC7901 cells, GES1 cells, and SGC7901/PA cells. B - CDC6 expression in BGC823, SGC7901, GES1 cells, and SGC7901/ PA cells. ${ }^{*} P<0.05$

A

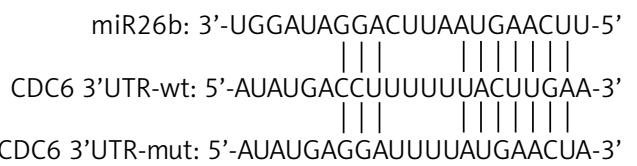

C

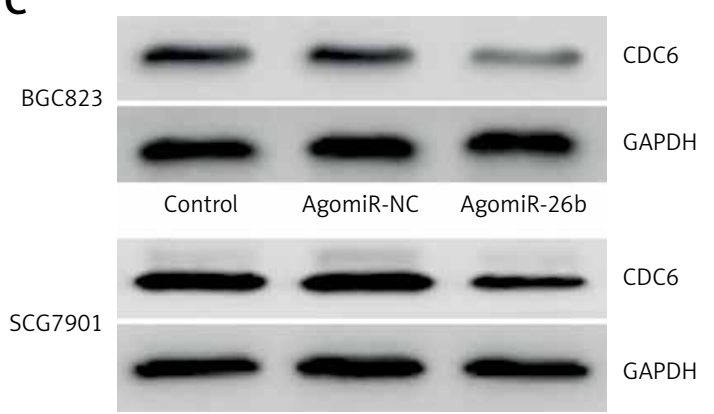

B

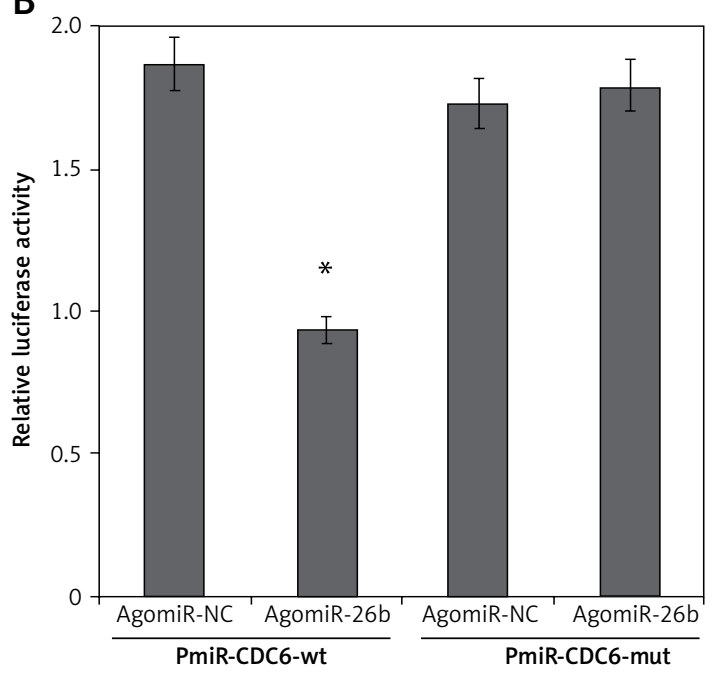

Figure 2. CDC6 is a target gene of miR-26b. A - 3'-UTR region of CDC6 mRNA is partially complementary to miR-26b. B - The relative luciferase activities in cells co-transfected with pmiR-CDC6-wt and agomiR-26b vs. control vector. Firefly luciferase activity was normalized to Renilla luciferase. C - Transfection of agomiR-26b suppressed the CDC6 protein level in BGC823 and SGC7901 cells. ${ }^{*} P<0.05$ 
A
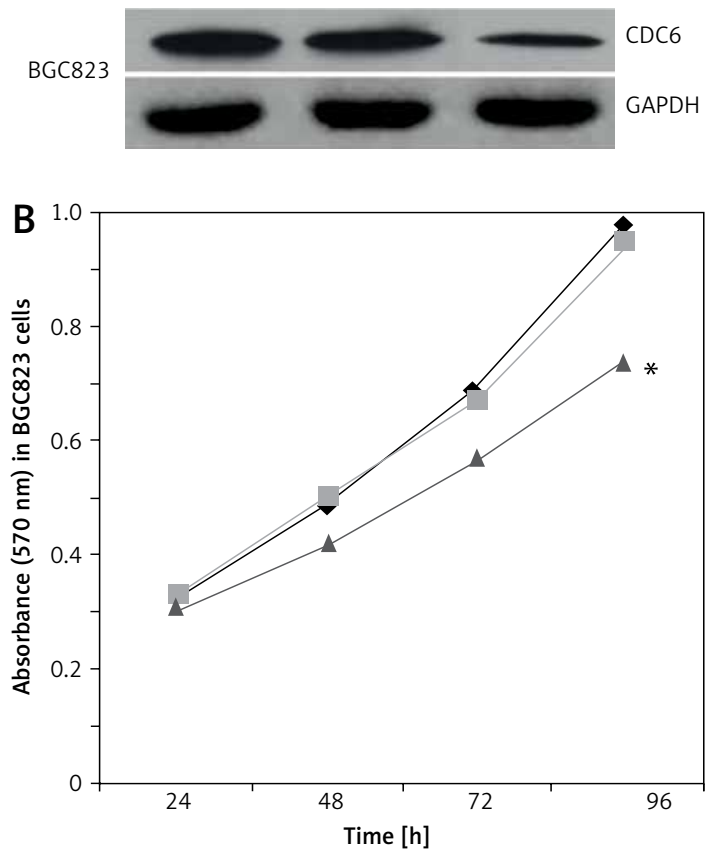

- Control $\rightarrow$ pS-NC $\quad$ - pS-CDC6

Control pS-NC pS-CDC6
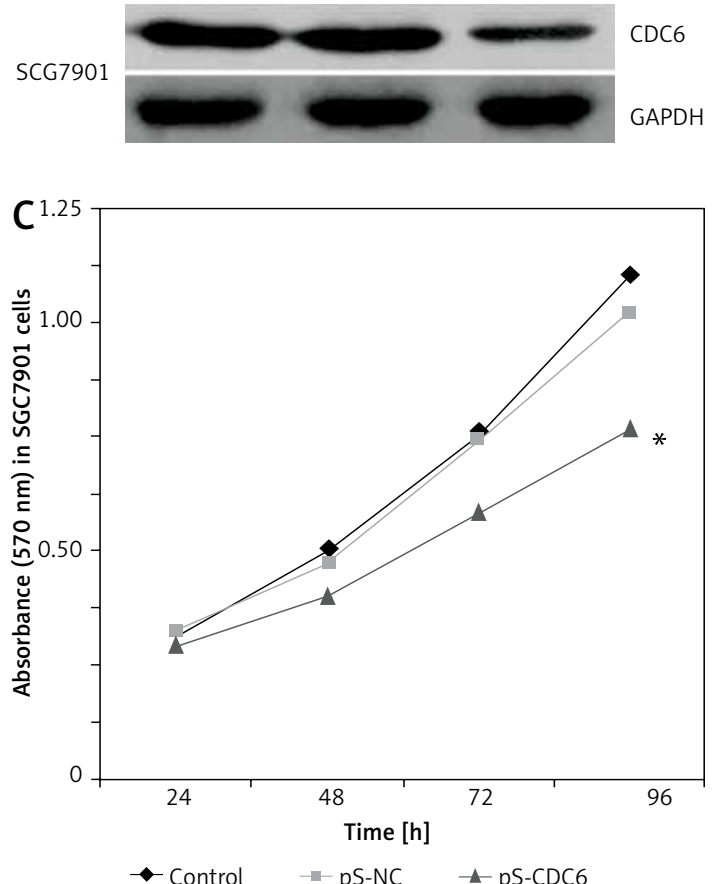

PS-NC pS-CDC6

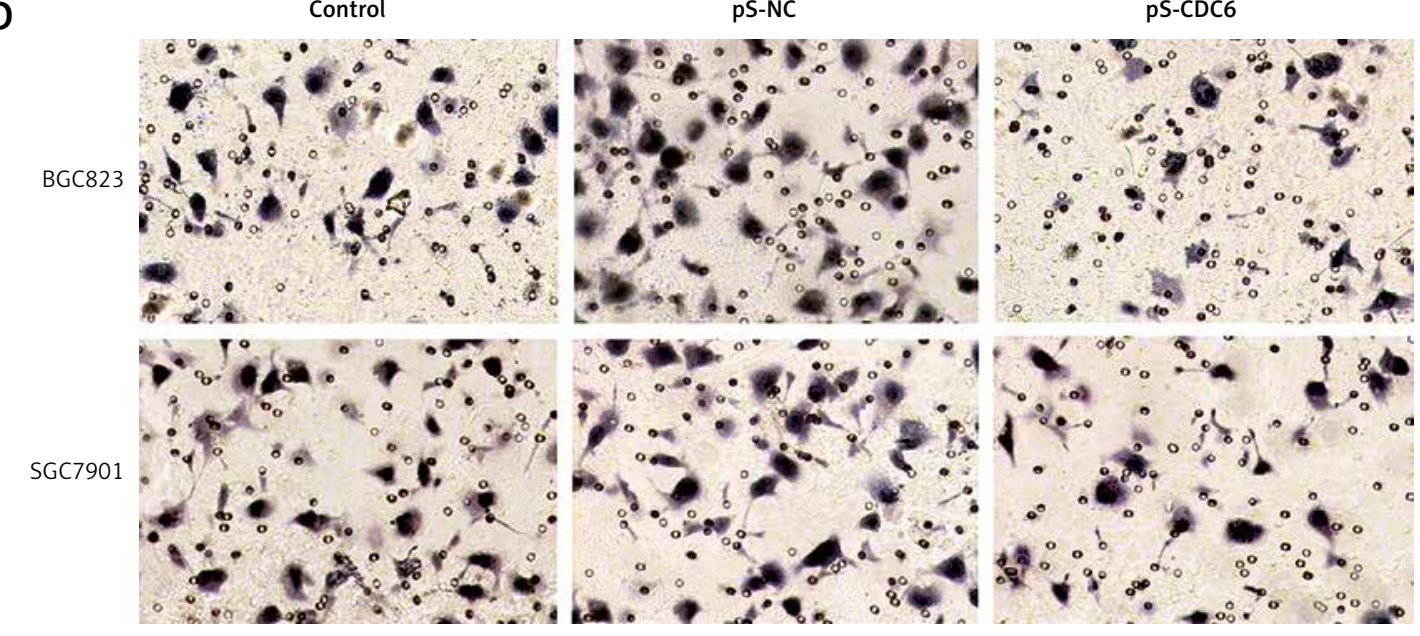

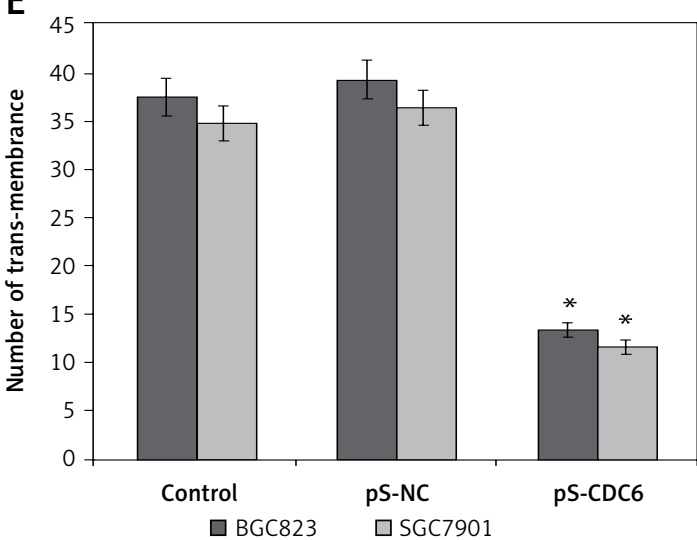

$\mathrm{F}$

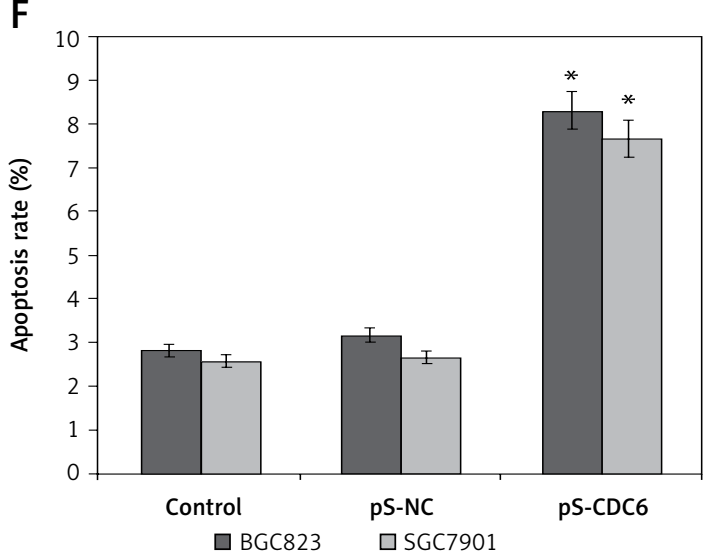

Figure 3. CDC6 knockdown inhibits the growth of GC cells. A - Transfection of pS-CDC6 knocked down CDC6 expression in BGC823 and SGC7901 cells. B, C - CDC6 knockdown significantly inhibited cellular proliferation of BGC283 (B) and SGC7901 (C) cells. D, E - CDC6 knockdown inhibited invasion of BGC283 and SGC7901 cells. F-CDC6 knockdown promoted cell apoptosis of BGC283 and SGC7901 cells. ${ }^{*} P<0.05$ 


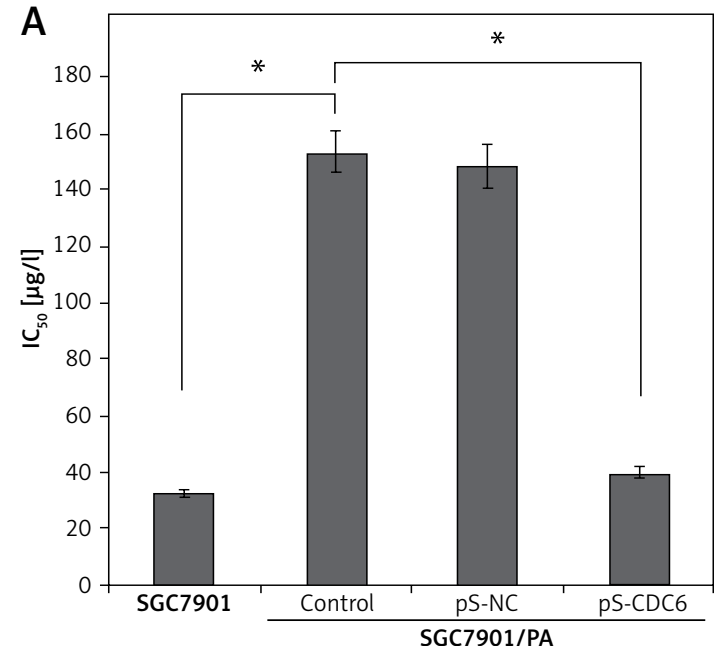

Consistent with the downregulated miR-26b level, CDC6 was highly expressed both in BGC823 and SGC7901 cells, in sharp contrast with GES1 cells. In addition, the expression of CDC6 was even higher in SGC7901/PA cells (Figure 1 B). By Pearson's correlation analysis, a negative correlation was identified between miR-26b and CDC6 $(p<0.05)$

We next used luciferase reporter assay to investigate whether CDC6 is a direct target of miR-26b. Compared to NC, the relative luciferase activity was significantly lower in HEK293T cells co-transfected with wild-type luciferase vector and agomiR-26b $(p<0.05)$ (Figure 2 B). These results suggest that miR-26b specifically binds the 3'UTR of the CDC6 gene to inhibit its expression. This was further confirmed by western blot at the protein level: CDC6 levels were significant lower in BGC823 and SGC7901 cells transfected with agomiR-26b (Figure $2 \mathrm{C}$ ). Taken together, the findings confirm that miR-26b negatively regulates endogenous CDC6 expression.

\section{CDC6 knockdown inhibited the growth and invasion of GC cells}

To ascertain whether CDC6 knockdown may affect the cell growth of GC, we first established BGC823 and SGC7901 cells overexpressing the silencing vector pS-CDC6, and confirmed that the expression levels of CDC6 protein were significantly lower, compared to cells transfected with control vectors ( $p<0.05$, Figure $3 A$ ).

As shown in Figure $3 \mathrm{~B}$ and $\mathrm{C}, \mathrm{CDC} 6$ knockdown inhibited cellular viability of both BGC823 and SGC7901 cells $(p<0.05)$. By using Transwell assay, we further found that CDC6 knockdown in BGC823 and SGC7901 cells significantly inhibited cell invasion (Figure $3 \mathrm{D}, p<0.05$ ). This likely was due to increased apoptosis in these cells, as shown in Figure $3 \mathrm{E}(p<0.05)$.

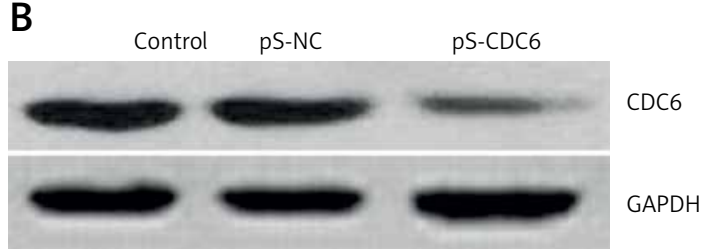

Figure 4. $C D C 6$ knockdown inhibited resistance to paclitaxel chemotherapy in SGC7901/PA cells. A - The paclitaxel concentration of $50 \%$ inhibition of cell growth (IC ${ }_{50}$ ) of SGC7901/PA cells was higher than that in SGC7901 cells, CDC6 silence decreased IC50 of paclitaxel in SGC7901/PA cells. B - Transfection of pS-CDC6 knockdown the CDC6 expression in SGC7901/ PA cells. ${ }^{*} P<0.05$

\section{CDC6 knockdown inhibited resistance to paclitaxel chemotherapy in SGC7901/PA cells}

Paclitaxel is widely used in patients with advanced GC. The half maximal inhibitory concentrations $\left(I C_{50}\right)$ of paclitaxel in SGC7901 and SGC7901/PA cells were $32.64 \pm 0.25 \mu \mathrm{g} / \mathrm{l}$ and $153.17 \pm 0.49 \mu \mathrm{g} / \mathrm{l}$, respectively; the calculated resistance index (RI) was 4.69. SGC7901 cells were significantly more sensitive to paclitaxel $(p<0.05$, Figure $4 \mathrm{~A})$. The expression of CDC6 was silenced in SGC7901/PA cells by transfecting pS-CDC6 ( $p<$ 0.05 , Figure $4 \mathrm{~B})$. This led to increased $I C_{50}$ of paclitaxel from $153.17 \pm 0.49 \mu \mathrm{g} / \mathrm{l}$ to $39.81 \pm 0.28 \mu \mathrm{g} / \mathrm{l}$ $(p<0.05$, Figure 4 A). The results suggest that silencing of CDC6 inhibited resistance to paclitaxel chemotherapy.

\section{Discussion}

Gastric cancer is an aggressive cancer that often is associated with resistance to chemotherapy or radiation [17-19]. This may have contributed to the high relapse rate of GC. Our findings of the downregulation of miR-26b in the GC cell lines suggest that miR-26b might serve as a tumor suppressor gene. This finding needs to be validated in GC tissue specimens. Moreover, miR-26b may also play a role in chemotherapy resistance, as its level was even lower in cells that are resistant to paclitaxel.

Overexpression of CDC6 has been reported in a variety of cancers $[20,21]$. CDC6 is an essential initiation protein for DNA replication, playing a vital role in tumorigenesis by regulating cell cycle, apoptosis, and invasion [22, 23]. In addition, it was reported to be involved in chemotherapy resistance by activation of the ATR-Chk 1 pathway [24]. Our findings of overexpression of CDC6 in the GC cell lines suggest that it may play a role in 
the tumorigenesis in GC. Moreover, it might also be involved in the development of resistance to paclitaxel chemotherapy in GC. If this finding can be generalized to GC tissue specimens, then targeting CDC by overexpressing miR-26b may serve as a promising anticancer strategy for GC.

\section{Acknowledgments}

This study was supported by the National $\mathrm{Na}$ ture Science Foundation of China (81272716) and Liaoning social development research plan (2012225016) and Shenyang science and technology project project (17-231-1-49).

\section{Conflict of interest}

The authors declare no conflict of interest.

\section{References}

1. Sugano K. Screening of gastric cancer in Asia. Best Pract Res Clin Gastroenterol 2015; 29: 895-905.

2. Newton AD, Datta J, Loaiza-Bonilla A, Karakousis GC, Roses RE. Neoadjuvant therapy for gastric cancer: current evidence and future directions. J Gastrointest Oncol 2015; 6: 534-43.

3. Lan G, Yang L, Xie X, Peng L, Wang Y. MicroRNA-490-5p is a novel tumor suppressor targeting c-FOS in human bladder cancer. Arch Med Sci 2015; 11: 561-9.

4. Chen J, Liu L, Liu Y, et al. Low-dose endothelial-monocyte-activating polypeptide-II induced autophagy by Down-regulating miR-20a in U-87 and U-251 glioma cells. Front Cell Neurosci 2016; 10: 128.

5. Zhao JJ, Chen PJ, Duan RQ, Li KJ, Wang YZ, Li Y. miR-630 functions as a tumor oncogene in renal cell carcinoma. Arch Med Sci 2016; 12: 473-8.

6. Tian L, Shan W, Zhang Y, Lv X, Li X, Wei C. Up-regulation of miR-21 expression predicate advanced clinicopathological features and poor prognosis in patients with non-small cell lung cancer. Pathol Oncol Res 2016; 22: 161-7.

7. Jin Q, Li XJ, Cao PG. MicroRNA-26b enhances the radiosensitivity of hepatocellular carcinoma cells by targeting EphA2. Tohoku J Exp Med 2016; 238: 143-51.

8. Lin J, Zhang L, Huang H, et al. MiR-26b/KPNA2 axis inhibits epithelial ovarian carcinoma proliferation and metastasis through downregulating OCT4. Oncotarget 2015; 6: 23793-806.

9. Kato M, Goto Y, Matsushita R, et al. MicroRNA-26a/b directly regulate La-related protein 1 and inhibit cancer cell invasion in prostate cancer. Int J Oncol 2015; 47: 710-8.

10. Du JY, Wang LF, Wang Q, Yu LD. miR-26b inhibits proliferation, migration, invasion and apoptosis induction via the downregulation of 6-phosphofructo-2-kinase/ fructose-2,6-bisphosphatase-3 driven glycolysis in osteosarcoma cells. Oncol Rep 2015; 33: 1890-8.

11. Zhang J, Yao T, Wang Y, Yu J, Liu Y, Lin Z. Long noncoding RNA MEG3 is downregulated in cervical cancer and affects cell proliferation and apoptosis by regulating miR21. Cancer Biol Ther 2016; 17: 104-13.

12. Zhu WS, Tang CM, Xiao Z, et al. Targeting EZH1 and $\mathrm{EZH} 2$ contributes to the suppression of fibrosis-associated genes by miR-214-3p in cardiac myofibroblasts. Oncotarget 2016; 7: 78331-42.
13. Shang C, Guo Y, Hong Y, Xue YX. Long non-coding RNA TUSC7, a target of miR-23b, plays tumor-suppressing roles in human gliomas. Front Cell Neurosci 2016; 10: 235

14. Cai D, Qiu Z, Yao W, et al. YSL-12, a novel microtubule-destabilizing agent, exerts potent anti-tumor activity against colon cancer in vitro and in vivo. Cancer Chemother Pharmacol 2016; 77: 1217-29.

15. Gu KS, Chen Y. Mechanism of P-glycoprotein expression in the SGC7901 human gastric adenocarcinoma cell line induced by cyclooxygenase-2. Asian Pac J Cancer Prev 2012; 13: 2379-83.

16. He T, Zhou H, Li C, et al. Methylglyoxal suppresses human colon cancer cell lines and tumor growth in a mouse model by impairing glycolytic metabolism of cancer cells associated with down-regulation of c-Myc expression. Cancer Biol Ther 2016; 17: 955-65.

17. Yang $\mathrm{Y}$, Jiang $\mathrm{M}, \mathrm{Hu}$ J, et al. Enhancement of radiation effects by ursolic acid in BGC-823 human adenocarcinoma gastric cancer cell line. PLoS One 2015; 10: e0133169.

18. Xu HB, Shen FM, Lv QZ. Celecoxib enhanced the cytotoxic effect of cisplatin in chemo-resistant gastric cancer xenograft mouse models through a cyclooxygenase-2-dependent manner. Eur J Pharmacol 2016; 776 : $1-8$.

19. Lee SY, Oh SC. Changing strategies for target therapy in gastric cancer. World J Gastroenterol 2016; 22: 1179-89.

20. Deng Y, Jiang L, Wang Y, et al. High expression of CDC6 is associated with accelerated cell proliferation and poor prognosis of epithelial ovarian cancer. Pathol Res Pract 2016; 212: 239-46.

21. Búa S, Sotiropoulou P, Sgarlata C, et al. Deregulated expression of CDC6 in the skin facilitates papilloma formation and affects the hair growth cycle. Cell Cycle 2015; 14: 3897-907.

22. Chen S, Wan P, Ding W, et al. Norcantharidin inhibits DNA replication and induces mitotic catastrophe by degrading initiation protein CDC6. Int J Mol Med 2013; 32: 43-50.

23. Zhang X, Xiao D, Wang Z, et al. MicroRNA-26a/b regulate DNA replication licensing, tumorigenesis, and prognosis by targeting CDC6 in lung cancer. Mol Cancer Res 2014; 12: $1535-46$.

24. Chen S, Chen X, Xie G, et al. CDC6 contributes to cisplatin-resistance by activation of ATR-Chk1 pathway inbladder cancer cells. Oncotarget 2016; 7: 40362-76. 